\title{
Two cases of Saint Louis encephalitis in HIV-1 infected patients in Buenos Aires
}

\section{ABSTRACT}

Saint Louis encephalitis virus (SLE) is a mosquito borne disease. Only a small proportion of cases progress to severe clinical forms. There have been few reports on HIV-infected patients and the relationship between immunodeficiency and the course of the disease remains unclear. Herein we describe two cases of SLE in HIV-1-infected patients in Buenos Aires city.

Keywords: HIV encephalitis; arbovirus; encephalitis virus, St Louis.

Saint Louis encephalitis (SLE) is a mosquitoborne disease caused by a member of the Flaviviridae family. Less than $1 \%$ of SLE viral infections are clinically apparent. Poor prognosis is associated with advanced age, and with higher frequency of meningoencephalitis. ${ }^{1}$ Although it has been responsible for several outbreaks in the general population, there has been few reports on HIV patients. ${ }^{2,3}$ The potential impact of HIV-1 induced immune deficiency over the SLE clinical course remains unclear. In Argentina, despite of a seroprevalence ranging from $3 \%$ to $50 \%,{ }^{4,5}$ only two outbreaks of SLE have been reported. ${ }^{6,7}$ Herein we describe two cases of meningoencephalitis caused by SLE virus in HIV-1-infected patients from Buenos Aires.

On late summer 2010, a 30-year-old man was seen at the emergency room because of fever and altered mental status. He had been diagnosed with HIV infection since 2007. He had never received antiretroviral therapy (HAART). He lived in Buenos Aires and had not travelled in the last year. There was no history of exposure to animals or contact with ill people. On admission he was disoriented and lethargic. Neck stiffness without focal neurologic signs was found. Laboratory findings were not remarkable. CD4+ cell count was 423 cells $/ \mathrm{mm}^{3}$. Computed tomographic scan of the brain was normal. Lumbar puncture showed mild mononuclear pleocytosis. PCR testing in cerebral spinal fluid (CSF) for HSV, enterovirus, and cytomegalovirus were nega- tive. Three days later, the patient remained febrile and meningeal signs had progressed. $\mathrm{He}$ became more lethargic, with incomprehensible speech and myoclonies. A magnetic resonance imaging (MRI) of the brain with gadolinium and an electroencephalogram showed no abnormalities.

On the fifth day, the patient clinical picture began to improve. He was discharged on the twelfth day with resolved clinical symptoms, totally oriented; only acute memory disturbances persisted.

One month later, a 44 year-old male patient was admitted with headache and confusion. HIV-1 infection had been diagnosed five months before. His baseline CD4 was 500 cells $/ \mathrm{mm}^{3}$ (24\%). No HAART was started. His medical record was unremarkable. On admission, he was febrile and confuse, with mild somnolence and occipital headache. Laboratory findings were unremarkable; CD4+ cell count was 402 cells $/ \mathrm{mm}^{3}$. A lumbar puncture was performed with mild leucocytosis (mononuclear cells). Electroencephalogram showed diffuse abnormal slow rhythm. On the fourth day, the patient developed transient aphasia, and a confusional syndrome became more evident. MRI with gadolinium revealed increased T2 and FLAIR signal involving both temporal lobes. PCR for enterovirus, HZV and HSV were negative at CSF. Mental status improved progressively, and fever disappeared. He was discharged on the twelfth day fully recovered.
Authors

Guillermo Alberto

Viloria $^{1}$

Mariana Angelica Kundro $^{2}$

Javier Jose Toibaro ${ }^{3}$ Alfredo Seijo ${ }^{4}$

Marcelo Horacio Losso

${ }^{1} \mathrm{MD}$; HIV Unit, Hospital JM Ramos Mejia, Buenos Aires, Argentina ${ }^{2} \mathrm{MD}$; HIV/AIDS

Fellowship, Hospital JM Ramos Mejia, Buenos Aires, Argentina

${ }^{3} \mathrm{MD}$; Staff, HIV Unit, Hospital JM Ramos Mejia, Buenos Aires, Argentina ${ }^{4} \mathrm{MD}$; Zoonosis Unit Head, Hospital F. J. Muñiz, Buenos Aires, Argentina ${ }^{5} \mathrm{MD}$; HIV Unit Head, Hospital JM Ramos Mejia, Buenos Aires, Argentina

Submitted on: 03/31/2011 Approved on: 04/29/2011

Correspondence to: Guillermo Alberto Viloria Urquiza 609 - C1221ADC Buenos Aires, Argentina gviloria@hivramos.org.ar

We declare no conflict of interest.

(C2011 Elsevier Editora Ltda. All rights reserved. 
Serum and CSF to determine SLE IgM antibodies were positive by MAC ELISA in both patients. We also performed neutralization tests (NT) in paired samples of serum for SLE to confirm diagnosis. Because of serologic cross reactions with other closely related flavivirus circulating in our country, NT were also performed against dengue and West Nile viruses. Both results were negative. Ink smear, Ziehl-Nielsen and Gram staining in CSF were negative. Fungal, bacterial, and mycobacterial CSF and blood cultures showed no growth.

To the best of our knowledge, this is the first report of SLE in patients with HIV infection in Buenos Aires, Argentina. The clinical course was benign with mild memory disturbances. It is important to highlight that SLE virus is an emerging pathogen with potential to spread rapidly, and should be considered in the differential diagnosis of CNS disease in HIV-infected patients living in Buenos Aires.

\section{REFERENCES}

1. Vaughn DW, Barrett A, Solomon T. Flavivirus In: Mandell: Mandell, Douglas, and Bennetts Principles and Practice of Infectious Diseases, 7th ed. Churchill Livingstone. Elsevier; 2009; 142:1714-36.

2. Okhuysen PC, Crane JK, Pappas J. St. Louis encephalitis in patients with human immunodeficiency virus infection. Clin Infect Dis. 1993; 17:140-1.

3. Wasay M, Diaz-Arrastia R, Suss RA, et al. St Louis encephalitis: a review of 11 cases in 1995 Dallas, Texas. Arch Neurol. 2000; 57:114-8.

4. Spinsanti LI, Ré V, Diaz MP, et al. Age-related seroprevalence study for St. Louis encephalitis in a population from Cordoba, Argentina. Rev Inst Med Trop. São Paulo 2002; 44:59-62.

5. Spisanti L, Re V, Basualdo M, et al. Seroprevalencia de la infección por el virus Encefalitis San Luis en la provincia de Formosa. Medicina (B Aires). 2000; 60:474-6.

6. Spisanti L, Diaz L, Glaistein N, et al. Human outbreak of St. Louis Encephalitis detected in Argentina, 2005. J ClinVirol. 2008; 42:27-33.

7. The Pan American Health Organization, Epidemiological Alert: Saint Louis Encephalitis, Risk of Viral Circulation, 24 March 2010. Available from: http://new.paho.org/hq/index. php? option $=$ com_content $\&$ task $=$ view\&id=2679\&Itemid $=1167$. [cited 2010 March 30]. 